\title{
Pesquisa
}

\section{Satisfação dos Usuários com os Serviços Públicos Odontológicos: comparando QASSaB e intrumento do PMAQ-CEO}

\author{
Users Satisfaction with Public Dental Services: comparing QASSaB and PMAQ- \\ CEO instrument
}

Thaynná Barboza Bezerra de Lima1; Wilton Wilney Nascimento Padilha²

${ }^{1}$ Mestranda do Programa de Pós-Graduação em Odontologia da UFPB, João Pessoa, PB, Brasil. 2Professor do Programa de Pós-Graduação em Odontologia da UFPB, João Pessoa, PB, Brasil.

Autor e endereço para correspondência:

Thaynná Barboza Bezerra de Lima, Programa de Pós-Graduação em Odontologia, UFPB Universidade Federal da Paraíba, Cidade Universitária, S/N, Castelo Branco III, João Pessoa PB, 58051-085, thaynna_bbl@hotmail.com.

\begin{abstract}
Resumo
Introdução: Satisfação do paciente é uma das dimensões envolvidas no cuidado ao usuário dos serviços de saúde. Porém ainda é pouco avaliada. Pode ser entendida como uma relação entre percepção e expectativa, ou seja, o que o paciente "viu" e o que ele esperava "ver". Objetivo: Comparar as dimensões presentes nos questionários mais utilizados em estudos de satisfação (QASSaB) e as dimensões existentes no instrumento de satisfação do PMAQ-CEO. Metodologia: Trata-se de um estudo comparativo e descritivo entre as dimensões do QASSaB e do PMAQ-CEO. Resultados: Foram analisados princípios importantes para o funcionamento do serviço público odontológico, sendo eles: justiça, beneficência, acolhimento, eficiência, eficácia, equidade e aceitabilidade. O Instrumento do PMAQ-CEO não abrange a totalidade destes princípios. O QASSaB abrange, mas muitas vezes, só com um questionamento, o que se torna impreciso. Conclusão: Nos dois questionários analisados não existem dimensões iguais, existem algumas questões semelhantes. O QASSaB é o que abrange maior quantidade de perguntas relacionadas a princípios importantes para o serviço.
\end{abstract}

Descritores: Satisfação do Paciente. Qualidade de Vida. Sistema Único de Saúde. 


\begin{abstract}
Introduction: Patient satisfaction is one of the dimensions involved in the care of the health services user. But it is still poorly evaluated. It can be understood as a relation between perception and expectation, that is, what the patient "saw" and what he hoped to "see". Objective: To compare the dimensions present in the most used questionnaires in satisfaction studies (QASSaB) and the existing dimensions in the PMAQ-CEO satisfaction instrument. Methodology: This is a comparative and descriptive study between the dimensions of the QASSaB and the PMAQ-CEO. Results: Important principles for the functioning of the public dental service were analyzed, such as: justice, beneficence, reception, efficiency, effectiveness, fairness and acceptability. The PMAQCEO Instrument does not cover all of these principles. The QASSaB covers, but often only with a questioning, which becomes minimal. Conclusion: In the two questionnaires analyzed there are no equal dimensions, there are some similar questions. The QASSaB is the one that covers a greater amount of questions related to principles.
\end{abstract}

Key-words: Patient Satisfaction. Qualityof Life. Unified Health System.

\title{
Introdução
}

A Constituição de 1988 define a Saúde como direito de todos e dever do Estado, ou seja, a partir deste documento o SUS deveria oferecer um serviço de qualidade para a população, organizando suas diretrizes para tornar o usuário satisfeito com o serviço recebido ${ }^{1}$. Satisfação do paciente é uma das dimensões envolvidas no cuidado ao usuário dos serviços de saúde. Porém ainda é pouco avaliada ${ }^{2}$. Pode ser entendida como uma relação entre percepção e expectativa, ou seja, o que o paciente "viu" e o que ele esperava "ver"3.

É importante que o usuário deixe de ser um sujeito passivo, e participe da análise do serviço utilizado por ele, para que assim o resultado do estudo seja o mais real possível ${ }^{4}$. É de extrema importância a realização de estudos de satisfação, sob a ótica do usuário, porém existe uma carência de instrumentos validados para serem usados nestas pesquisas, principalmente no âmbito da Odontologia. Nesta área, a maioria dos estudos são realizados na atenção básica, e em minoria na atenção secundária ${ }^{5}$.

Os instrumentos utilizados para estudos de avaliação da qualidade dos serviços devem avaliar, principalmente, aspectos não clínicos, que contam bastante na avaliação do serviço por parte do usuário, são eles: cordialidade e presteza no atendimento, segurança do profissional, organização e beleza do ambiente, informações e explicações fornecidas e qualidade das informações ${ }^{3}$. 
Um dos instrumentos validados mais utilizados em pesquisa de satisfação é o Questionário de Avaliação da Qualidade dos Serviços de Saúde Bucal (QASSaB), este instrumento foi validado em uma tese de doutorado, visando a uniformidade de instrumentos para estudos deste tipo, e foi baseado na proposta de Avaliação de Qualidade proposto por Donabedian ${ }^{3}$. O Programa de Melhoria do Acesso e da Qualidade (PMAQ), tanto o da Atenção Básica (PMAQ-AB) quanto o do Centro de Especialidades Odontológicas (PMAQ-CEO), possui um modelo de avaliação externa, que dentre os questionários, existe um que avalia a satisfação do usuário com o serviço. Pois, estudar satisfação faz parte das relações interpessoais no âmbito dos serviços de saúde, fortalecendo assim, a participação da comunidade nos processos de planejamento e avaliação dos serviços ${ }^{6}$.

O QASSaB abrange 10 dimensões, são elas: Disponibilidade dos Serviços, Resolutividade, Ambiente Físico do Serviço - Limpeza, Relações Humanas (Relações com Profissionais de Saúde), Relações Humanas (Relação Dentista/Paciente), Qualidade Técnico-Científica dos Equipamentos, Eficiência, Eficácia, Equidade e Aceitabilidade. O Instrumento do PMAQ-CEO com relação à satisfação apresenta as dimensões: Acesso aos Serviços do CEO, Marcação de Consultas neste CEO, Acolhimento, Atendimento, Vínculo e Responsabilização, Prótese Dentária, Insumos, Equipamentos e Ambiência. São poucos os estudos, na literatura, de comparação de questionários de satisfação com os serviços odontológicos.

Sendo assim, o objetivo deste estudo é comparar as dimensões presentes nos questionários mais utilizado em estudos de satisfação (QASSaB) e as dimensões existentes no instrumento de satisfação do PMAQ-CEO.

\section{Metodologia}

Trata-se de um estudo comparativo e descritivo entre as dimensões do QASSaB e do PMAQCEO. Todas as questões de cada instrumento foram tabuladas no programa Excel e comparadas. Foram analisadas as perguntas iguais, as dimensões que coincidiam e as perguntas diferentes presentes em cada uma. Além, da importância de cada dimensão presente em ambos os questionários.

A comparação foi feita através de princípios considerados importantes para o atendimento ao usuário, são eles: justiça, beneficência, acolhimento, eficiência, eficácia, equidade e aceitabilidade. 


\section{Resultados}

Foram descritos e comparados os princípios existentes no QASSaB e no PMAQ-CEO (Quadro 1). Alguns se assemelham nos dois questionários, enquanto outros princípios não estão presentes no PMAQ-CEO.

\begin{tabular}{|c|c|c|}
\hline \multicolumn{3}{|c|}{ Quadro 1. Descrição dos Princípios presentes no QASSaB e PMAQ-CEO } \\
\hline Princípios & QASSaB & PMAQ-CEO \\
\hline Justiça & $\begin{array}{l}\text { Dimensão "Disponibilidade dos Serviços" ( } 3 \\
\text { questões) }\end{array}$ & $\begin{array}{l}\text { Presente (1 questão - Dimensão Acesso aos } \\
\text { Serviços do CEO) }\end{array}$ \\
\hline Beneficência & Dimensão "Resolutividade" (3 questões) & $\begin{array}{l}\text { Presente (1 questão - Dimensão Marcação de } \\
\text { Consultas no CEO) }\end{array}$ \\
\hline Acolhimento & Dimensão “Relações Humanas" (5 questões) & Presente (1 questão - Dimensão Acolhimento) \\
\hline Eficiência & Dimensão "Eficiência" (1 questão) & Ausente \\
\hline Eficácia & Dimensão "Eficácia” (1 questão) & Ausente \\
\hline Equidade & Dimensão "Equidade" (1 questão) & Ausente \\
\hline Aceitabilidade & Dimensão "Aceitabilidade" (2 questões) & $\begin{array}{l}\text { Presente (2 questões, mesma quantidade de } \\
\text { QASSaB - Dimensão Vínculo e } \\
\text { Responsabilização) }\end{array}$ \\
\hline
\end{tabular}

*PMAQ-CEO (Programa de Melhoria do Acesso e da Qualidade dos Centros de Especialidades Odontológicas; QASSaB- Questionário de Avaliação da Satisfação dos Usuários com os Serviços de Saúde Bucal

\section{Discussão}

Justiça é a particularidade do que é justo e correto, como o respeito à igualdade de todos os cidadãos. Em Saúde, a justiça está relacionada à equidade na distribuição de bens e de benefícios na área de saúde ${ }^{7}$. No Instrumento do QASSaB há uma dimensão que representa este princípio, a dimensão "Disponibilidade dos Serviços", possui 3 questões e se preocupa em saber se o paciente obteve vaga no serviço facilmente, o tempo de marcação entre uma consulta e outra, e o tempo de espera para ser atendido. No Instrumento do PMAQ-CEO existe uma questão que se pode dizer condizente com este princípio ("Quanto tempo o Senhor esperou para iniciar o tratamento neste CEO?"), presente na Dimensão “Marcação de Consultas neste CEO”.

Beneficência é considerado o comportamento da pessoa que busca ajudar ou fazer bem ao próximo. Na área da Saúde ocupa-se da busca do bem-estar do paciente, evitando danos na 
medida do possível7. Com relação a este princípio, o QASSaB possui a dimensão "Resolutividade" com 3 perguntas que pergunta se o problema do paciente foi resolvido e se ele está satisfeito com a aparência dos dentes tratados. O Instrumento do PMAQ-CEO aborda na dimensão "Marcação de Consultas", uma questão sobre este aspecto (Quanto ao problema que levou o Senhor a ser atendido neste CEO, classificaria como).

Acolhimento é uma diretriz da Política Nacional de Humanização $(\mathrm{PNH})$, que não tem local nem hora certa para acontecer, nem um profissional específico para fazê-lo: faz parte de todos os encontros do serviço de saúde. É a humanização do atendimento, isto é, das relações entre trabalhadores e serviços de saúde com seus usuários ${ }^{8}$. É um fator importante na busca pela satisfação do usuário com o serviço, já que é papel da ESF promover a humanização com os usuários, estimulando assim o reconhecimento de saúde como questão de cidadania ${ }^{9}$. O QASSaB em suas dimensões referentes às Relações Humanas (Profissionais de Saúde/Cirurgião-Dentista) aborda 5 questões que se relacionam com o princípio citado, pois procura saber a atenção que os profissionais disponibilizaram ao paciente durante o atendimento. O Instrumento do PMAQ-CEO aborda uma questão na dimensão "Acolhimento" ("De zero a dez, qual nota o Senhor atribui para sua satisfação com o cuidado recebido pelo dentista?").

A eficiência é definida como a qualidade daquilo ou de quem é competente, que realiza de maneira correta as suas funções. O QASSaB destina uma dimensão para este princípio, porém com apenas uma questão. O PMAQ-CEO não aborda este princípio.

Eficácia é a qualidade daquilo que cumpre com as metas planejadas, ou seja, uma característica pertencente aos serviços que alcançam os resultados esperados. É importante este princípio em estudos que se propõem a avaliar a qualidade dos serviços. O QASSaB destina uma Dimensão específica para este princípio, com cinco questões. O Instrumento do PMAQ-CEO, não contempla estes princípios em suas avaliações.

O conceito do princípio da equidade revela o uso da imparcialidade para reconhecer o direito de cada um, usando a equivalência para se tornarem iguais, ou seja, oferecer mais, a quem necessita de mais. Todo o SUS necessita ser equânime. Sabendo disto, o Instrumento de avaliação QASSaB, disponibiliza uma dimensão para este ponto, com uma questão. Já o PMAQ-CEO não aborda este princípio em sua avaliação externa.

Em Saúde, o princípio da aceitabilidade está associado à aceitação do serviço que é oferecido, pelos usuários. O QASSaB possui uma dimensão com este nome, com duas questões. O PMAQ-CEO também aborda esta dimensão com duas questões, uma está inserida na Dimensão "Vínculo e Responsabilização" ("Durante o tratamento, o dentista esclareceu sobre seu 
tratamento?") e a outra pergunta está inserida na Dimensão "Marcação de Consultas" (“O Senhor foi consultado sobre melhor horário e dia para agendar seu atendimento?").

No QASSaB ainda há a abordagem da Qualidade Técnico-Científica dos Equipamentos e do Ambiente Físico do Serviço/Limpeza. Com perguntas relacionadas à tecnologia dos equipamentos utilizados e relacionadas à limpeza de ambientes como recepção, consultório e banheiros. Em comum com o PMAQ-CEO apenas o questionamento sobre o conforto do paciente no local do serviço. Este último Instrumento possui um diferencial de abordagem de uma dimensão exclusiva para Prótese Dentária.

\section{Conclusão}

Nos dois questionários analisados não existem dimensões iguais, existem algumas questões semelhantes. O QASSaB é o que abrange uma maior quantidade de perguntas relacionadas a princípios importantes para o serviço.

\section{Referências}

1. Mialhe FL, Oliveira CSR, Silva DD. Evaluation of the acccess to dental care and oral health services in a rural area of southern Brazil. Arq. Ciênc. Saúde Unipar. 2006; 10(3):145-149.

2. Suda EY, Uemura MD, Velasco E. Avaliação da satisfação dos pacientes atendidos em uma clínica-escola de fisioterapia de Santo André, SP. Fisioterapia e Pesquisa. 2009;16(2):126131.

3. Castro JDB, Silva VB. Satisfação no Atendimento Odontológico: um estudo na COE (Clínica Odontológica de Ensino) de Anápolis - Unievangélica. Revista Administra-ação. 2008. 5(1):34-44.

4. Bottan ER, Sperb RAL, Telles PS, Uriarte Neto M. Avaliação de serviços odontológicos: a visão dos pacientes. Revista da ABENO. 2005; 6(2):128-133.

5. Lima ACS, Cabral ED, Vasconcelos MMVB. Satisfação dos usuários assistidos nos Centros de Especialidades Odontológicas do Município do Recife, Pernambuco, Brasil. Cad Saúde Pública. 2010; 26(5):991-1002. 
6. Protasio APL, Gomes LB, Machado LS, Valença AMG. Satisfação do usuário da Atenção Básica em Saúde por regiões do Brasil: 1ํ ciclo de avaliação externa do PMAQ-AB. Ciência \& Saúde Coletiva. 2017; 22(6):1829-1844.

7. Garbin CAS, Mariano RQ, Machado TP, Garbin AJI. Estudo Bioético das Relações Humanas no Tratamento Odontológico. Rev FOL/UNIMEP. 2002:14(1);54-59.

8. Carvalho CAP, Marsicano JA, Carvalho FS, Sales-Peres A, Bastos JRM, Sales-Peres SHC. Acolhimento aos usuários: uma revisão sistemática do atendimento no Sistema Único de Saúde. Arq Ciênc Saúde. 2008; 15(2):93-95.

9. Fadel CB, Sandrini JC, Zardo LN. Avaliação do perfil e grau de satisfação dos usuários dos serviços odontológicos na Estratégia Saúde da Família, na Zona Rural do município de Castro/PR. Publ. UEPG Ci. Biol. Saúde. 2007;13(4):59-66. 